früher von uns bestimmten Adeninnucleotide in der Leber dafür, daß es bis zur 4. Std. nach $\mathrm{CCl}_{4}$ noch nicht zu einer irreversiblen Schädigung gekommen ist (2).

Wir haben daher in einer zweiten Versucbsserie die Gesamtaktivität vor allem jener Enzyme in der Leber gemessen, deren Aktivität im Serum bei einer Leberschädigung speziell durch $\mathrm{CCl}_{4}$ typischerweise ansteigt. Die in den Abbildungen 1 und 2 dargestellten Ergebnisse bestätigen die Ergebnisse der ersten Versuchsreihe. Abgesehen von einer zeitlichen Verschiebung des Maximums, die durch das Tiermaterial bedingt sein mag, kommt es auch hier zu einer Aktivitätszunahme aller untersuchten Enzyme in der Leber, die Zunahme bis zur 4. Std. ist für Lactatdehydrogenase, Alanintransaminase und Malatdehydrogenase gegenüber den Kontrollen statistisch signifikant. Nach der 4. Std. fallen die Enzymaktivitäten dann deutlich und steil wieder ab.

Wir haben uns daraufhin die Frage nach der Ursache des Aktivitätsanstieges gestellt. Abgesehen davon, daß es unwahrscheinlich ist, daß so unterschiedliche En- zyme gleichzeitig durch eine Substanz oder einen Mechanismus aktiviert werden, spricht die signifikante Zunahme des löslichen Protein in der Leber 4 Stdn. nach $\mathrm{CCl}_{4}$ gegen eine Aktivierung und für eine $\mathrm{Zu}$ nahme des Enzymproteins im wäßrigen Extrakt. Eine endgültige Entscheidung ist auf Grund unserer bisherigen Befunde nicht möglich. Jedoch allein ein Enzymaktivitätsanstieg in der Leberzelle zu einem Zeitpunkt, da die gleichen Enzymaktivitäten im Serum bereits deutlich und signifikant erhöht sind, läßt sich unseres Erachtens nicht mit einer erhöhten Membrandurchlässigkeit erklären $(5,6)$. Es dürfte daher neben dem Enzymverlust auf Grund einer Schädigung der Zellmembran, wie er sicher für Zellnekrosen zutrifft, ein weiterer Mechanismus für den Anstieg der Serumenzyme existieren. Es ist die Frage, ob dieser Mechanismus, der unseres Erachtens auf Veränderungen der Enzyme im weitesten Sinne beruhen muß, spezifisch für die akute Tetrachlorkohlenstoffvergiftung ist oder auch $z$. B. bei der Virushepatitis wirksam wird.

Wir danken Fräulein Ursula GNEIST für fleißige und gewissenhafte Mitarbeit bei der Durchführung der Versuche.

\title{
Literatur
}

1. KRÖNER, H. und W. StAIB, diese Z. 5, 89 (1967). - 2. KRÖNER H. und W. Starb, Hoppe-Seyler's Z. physiol. Chem. 348, 575 (1967). - 3. Bruns, F. H., E. Brosswitz, H. DenNemanN, H. D. Horn und E. NoltmanN, Klin. Wschr. 39, 342 (1961). 4. Hess, B., Enzyme im Blutplasma, G. Thieme-Verlag, Stuttgart (1962). - 5. ZierLer, K. L., Amer. J. Physiol. 185, 1 (1956). 6. Ziercer, K. L., Ann. N. Y. Acad. Sc. 75, 227 (1958). - 7. Beaufay, H., E. v. Campenhout und C. De Duve, Biochem. J. 73, 617 (1959). - 8. De Duve, C., R. Wattraux und P. BaudHuns, Adv. Enzymol. 24, 291 (1962). - 9. Frshman, W. H., in: Methoden der enzymatischen Analyse, Hrsg. H. U. Bergmeyer, Verlag Chemie GmbH, Weinheim/Bergstraße (1962). - 10. Berthet, J. und C. DE Duve, Biochem. J. 50, 174 (1951). -
11. Hövels, O. Ztschr. für Kinderhk. 66, 237 (1949). - 12 HARper, A. E., in: Methoden der enzymatischen Analyse, Hrsg. H. U. Bergmeyer, Verlag Chemie GmbH, Weinheim/Bergstraße (1962). - 13. Martin, J. B. und D. M. Doty, zit. nach H. WeilMalherbe. In Hoppe-Seyler-Thierfelder Handbuch Physiol. Pathol Chem. Analyse III, 1, Hrsg. K. Lang und E. Lehnartz, Springer, Berlin, Göttingen, Heidelberg (1955). - 14. SteINer, J. W., M. J. Philips und K. Mryar, Internat. Rev. exp. Pathol. 3, 65 (1964). - 15. McLean, A. E. M. und E. K. McLean, Biochem. J. 100, 564 (1966). - 16. Slater, T. F. Nature London 209, 36 (1966). - 17. Bruns, F. H. und J. Neuhaus, Biochem. $Z$. 326,242 (1955).

Prof. Dr. W. Staib 4 Düsseldorf 1 Witzelstr. 111

\section{Die klinische Bedeutung der Porphyrien ${ }^{1}$ )}

\author{
Von J. BRUGSCH \\ Herrn Prof. Dr. Dr. Ernst Scbütte zum 60. Geburtstag gewidmet
}

(Eingegangen am 23. März 1968)

Es wird auf die Bedeutung und die Differenzierung verschiedener Porphyrien für den Menschen und ihre praktische Bedeutung hingewiesen.

The differentiation of various types of human porphyria, and their importance and practical significance are discussed.

Als wir 1933 bei Hugo KäMmerer in München und bei HANS Frscher Untersuchungen des Porphyrinstoffwechsels beim Menschen begannen, hatte bereits HaNs GüNTHER 1911 die Porpbyria cutanea congenita (1) und 1920 die akute Porphyrie beschrieben (2).

1) Vortrag gehalten im Juni 1967 auf dem V. Internationalen Symposium für Hepatologie in Terme di Chianciano/Italien.
Während Hugo Kämmerer (3) noch 1933 die Anschauung vertreten hatte, daß Porphyrine sowohl im Häminaufbau als auch -abbau entstehen könnten, ist es heute klar, daß die bei Porphyrien auftretenden Uroporphyrine I und III als Störungen der Hämsynthese aufzufassen sind. Wir wissen durch die Aufklärung der zellulären Hämsynthese einigermaßen über diese aus Succinat und Glykokoll erfolgende 
Synthese über $\alpha$ Amino - $\beta$ Ketoadipinsäure, $\delta$-Aminolaevulinsäure, Porphobilinogen und die Bildung der Uroporphyrine I und III aus Leuko-Verbindungen Bescheid. Die klinische Forschung hat, nachdem wir und andere die Möglichkeiten des Nachweises und der Bestimmung dieser Porphyrine auch im kleinsten Labor eingeführt hatten, auch in Deutschland, in dem die erste chemische Porphyrin- und Protohäminsynthese durch HaNs FisCher durchgeführt wurde, auch wieder ernst zu nehmende Forschungen bewirkt. Es hat sich folgendes ergeben, wobei wir unsere eigenen Erfahrungen hier in der Darstellung und Definition bevorzugen:

Wenn wir als echte Porphyrien Krankheitszustände bezeichnen, bei denen Uroporphyrine in großen Mengen gebildet, umgesetzt, abgelagert und auch ausgeschieden werden, kommen derartige Krankheitszustände, bei denen der Uroporphyrinnachweis demnach leicht gelingt, als angeborene oder als erworbene Krankheitsbilder zur Beobachtung. Es zeigt sich, daß es sich bei der "Porpbyria cutanea congenita" als Lichtdermatose um eine seltene Krankheitsform einer vorwiegend die Erythrocyten befallenden angeborenen Hämsynthesestörung mit Auftreten großer Uroporphyrinmengen in den Erythroblasten handelt. Aus diesem Grunde wurde von Schmid, Schwartz und Watson (4) diese Form der Porpbyria congenita Hans Günthers als Porpbyria erythropoietica bezeichnet.

Erst späterhin beobachtete man, daß beim Erwachsenen, ohne viel Anhaltspunkte für eine Vererbung des Krankheitsbildes, eine meist milder verlaufende Form vorkommt, die man vor allem als erworbene Krankheitsform der Hämsynthesestörung auf der Uroporphyrinstufe ansprechen muß. In diesen Fällen der Porpbyria cutanea tarda findet man Hauterscheinungen der Blasenbildung mit Hyperpigmentierung, Narbenbildung und Hypertrichose im allgemeinen oft weniger erheblich, aber vorwiegend an den dem Licht ausgesetzten Körperregionen. Schmid, Schwarz und Watson nannten dieses Krankheitsbild Porpbyria bepatica, da große Uroporphyrinmengen im Lebergewebe abgelagert nachweisbar sind, die durch Leberpunktion als Rotfluoreszenz im UV-Licht leicht erkannt werden. Diese Form der Lichtkrankheit des Erwachsenen ist vor allem den Hautärzten bekannt und wird, wenn die Hauterscheinungen „ekzemartig“ verlaufen, oft lange Zeit von den Ärzten der Praxis verkannt.

Eine weitere Form der hepatischen Porphyrie stellt die Melanodermie-Porphyrie dar, die wir selbst erstmalig 1955 beobachtet und beschrieben haben $(5,6)$. Hier handelt es sich um Kranke, bei denen keinerlei Lichtempfindlichkeit der Haut besteht, wohl aber eine auffallende Überpigmentierung der Haut, die an Hämochromatose erinnert und in der Vergangenheit offensichtlich (z. B. von EPPINGER (7)) mit Hämochromatose verwechselt wurde, zumal bei Porphyrie des Erwachsenen Diabetes mellitus nicht selten ist. Fassen wir die cutanen Porphyrien des Erwachsenen mit den Melanodermie-Porphyrien als bepatische Porpbyrien zu- sammen, haben wir bis 1961112 derartige Fälle hepatischer Porphyrien beobachtet, von denen 53 Fälle als Porpbyria cutanea tarda und 48 als MelanodermiePorphyrie bezeichnet wurden.

Die akuten Porphyrien von HaNs GüNTHER, später auch als intermittierende Porphyrien bezeichnet, sind nicht als echte Porphyrien gekennzeichnet, sondern verlaufen im allgemeinen als Praeporphyrien. Hierunter verstehen wir Krankheitsbilder, bei denen nicht Uroporphyrin, sondern solche Vorstufen vorwiegend bedeutungsvoll auftreten, die wie Porphobilinogen und Leuko-Uroporphyrin leicht in Uroporphyrin übergeführt werden können. Dies kann im Reagensglas, aber auch nachträglich im Harn vor sich gehen. Uroporphyrin wird bei der akuten Porphyrie demnach erst spät und im klassischen Anfall des an akuter Porphyrie Erkrankten deutlich. Wir können hier über 21 Fälle von akuter Porphyrie berichten. Über die Bedeutung genetischer Faktoren mit dominantem Vererbungsgang bei akuter Porphyrie hat schon WALDENSTRÖM 1937 eingehend berichtet (8). Neben der akuten Porphyrie als Praeporphyrie haben wir dann noch die Leukoporphyrie als eine Praeporphyrieform beschrieben, bei der vor allem Leuko-Uroporphyrin als Uroporphyrinogen, aber kein Porphobilinogen-Nachweis mit EHRLICHs Reagens möglich ist (9). Dieser Nachweis, der sonst so kennzeichnend für die akute Porphyrie ist und als Umkehrprobe (demnach 2 Tropfen Harn zu 20 Tropfen Aldehyd-Reagens) ausgeführt wird, ist erstmalig von WATSON angegeben worden und von uns vereinfacht und dringend vor allem für die operative Sektion der Medizin empfohlen worden. Bei allen unklaren abdominalen, mit heftigen Schmerzen bis zu Koliken einhergehenden Krankheitsbildern, ist stets an akute Porphyrie zu denken. Die lebensgefährlichen nervösen und psychischen Störungen entwickeln sich oft erst im Anschluß an die Operation und können durch den kleinen Test vermieden werden. Vor allem Evipannarkosen sind gefährlich, aber schon eine Luminalspritze kann auf das Äußerste gefährden. Bei Einsetzen der Lähmungen ist die Gefährdung der meist weiblichen Kranken außerordentlich groß. Hier ist als Therapie die Infusion großer Flüssigkeitsmengen als physiologische Salzlösungen das Mittel der Wahl und führt, wenn nicht zu spät vorgenommen, gewöhnlich zum Erfolge und damit zum langsamen Abklingen der Lähmungen. Kommt es aber zu Blutungen in die Stammganglien, ist jeder Erfolg versagt. $\mathrm{Da}$ Uroporphyrin bei der akuten Porphyrie in der Leber als Vorstufe vorliegt, hatte WATSON auch diese als Porphyria hepatica bezeichnet; meines Erachtens jedoch .nicht zu Recht, da freies Uroporphyrin dann in der Leber vorkommt, wenn es sich um Mischformen von Porphyria cutanea tarda mit Photodermatose oder Melanodermie-Porphyrie mit akuter Porphyrie handelt. $\mathrm{Zu}$ den eigentlichen Porphyria-bepatica-Fällen, demnach der Porphyria cutanea tarda und der MelanodermiePorphyrie sei folgendes bemerkt: Die Kranken sind, im Gegensatz zur akuten Porphyrie, meist männlichen 
Geschlechtes und im späten Mannesalter. Etwa die Hälfte aller Fälle bei uns zeigt eine Vorgeschichte, die mäßigen oder starken Alkoholmißbrauch erkennen läßt. Außerdem kann Medikamentengabe, vor allem Salvarsanbehandlung festgestellt werden. Zuweilen ist Hepatitis in der Vorgeschichte angegeben und chronische Hepatitis wird histologisch nachweisbar. Die Beziehung zwischen hepatischen Prophyrien und Diabetes mellitus ist sehr zu beachten. Etwa 20\% der Porphyriker sind Diabetiker. Histologisch ist Eisenreichtum des Pankreas von uns beobachtet und beschrieben worden (9). Das histologische Bild der Leber zeigt außer Porphyrie meist Verfettung, Eisenreichtum, intra- und extrazellulär, und Zirrhosetendenz. Der Eisengehalt kann aber auch gering sein. Oft, vor allem bei der Porphyria cutanea tarda ist der Eisengehalt des Blutes erhöht. Der Zirrhosetendenz entsprechend finden sich nicht selten Palmarerythem und zuweilen „Sternchen" der Haut. Bei Porphyria-hepatica-Fällen ist häufig eine Neigung zu Gefäßerkrankungen nachzuweisen, die mit Neigung zur Vermehrung der $\alpha_{2}$ Globulin-Fraktion bei der Serumelektrophorese einhergeht. Die Vermehrung der $\gamma$-Globuline kann zudem erheblich sein.

Zwischen Porpbyria cutanea tarda und MelanodermiePorpbyrie bestehen wichtige und klinisch bedeutsame Unterschiede. Die Melanodermie-Porphyrie mit ihrer Überpigmentierung an den belichteten Hautstellen kann in die Porphyria cutanea tarda übergehen, demnach als Vorstadium, das sich über Wochen und Monate erstreckt, verlaufen. Sie kann aber auch, wie wir dies öfter gesehen haben, akut zum Tode führen. Während der Verlauf der cutanen Porphyrie sich bei vernünftiger Schonung des Kranken als recht günstig erweist, finden wir den Kranken mit Melanodermie-Porphyrie erheblich gefährdet. Wir sahen Todesfälle mit $\mathrm{Me}-$ lanodermie-Porphyrie als schwere Infekte mit tödlichem Ausgang bei Grippe, dann aber auch durch Gefäßkomplikationen, Apoplexie und Herzinfarkt. Wir haben letzteres auch als ,schwarzen Infarkt" bezeichnet. Die Beziehung der Porphyriker zu Erkrankungen der Gefäße mit Dysbasie, Coronarsklerose und zerebraler Gefäßerkrankung, sowie Hypertonie ist klinisch sehr bedeutungsvoll, zumal man mit Úberempfindlichkeit gegen Medikamente zu rechnen hat. Wir selbst sind bei der Porphyria cutanea tarda bereit, die Prognose relativ günstig zu stellen, ohne daß wir die Aderlaß- therapie IpPENS verwenden. Wir haben eine große Zahl von solchen Porphyrikern Jahre und über ein Jahrzehnt behandelt, wobei allerdings die Neigung zur Carcinombildung bei Porphyrikern erwähnt werden muß. Bei der Melanodermie-Porphyrie ist leider der Tod im Coma hepaticum nicht selten, wobei man daran denken sollte, daß ein allgemeiner Zusammenbruch der Leberfunktionen sich auch als ,symptomatische Porphyrie" erweisen kann.

Zur Diagnosenstellung sei noch folgendes bemérkt. Der Nachweis großer Uroporphyrinmengen im Harn ist und bleibt von entscheidender Bedeutung für die Porphyriediagnose. Hierzu hat sich der von uns als Filterverfahren mit Bleiacetatadsorption 1949 angegebene Uroporphyrinnachweis (10) überall bewährt. Die Bedeutung der Porphyriediagnose liegt meines Erachtens darin, daß man wohl mit Besserung - vor allem bei der Porphyria cutanea tarda und bei der akuten Präporphyrie und der meist durch Bleivergiftung ausgelösten Leukoporphyrie - rechnen kann, kaum aber mit Heilungen. Die Entwicklung einer MelanodermiePorphyrie ist oft ungünstig und muß daher durch Verhinderung jeglicher Leberschädigung möglichst. vermieden werden; der Zusammenbruch der Leberfunktion bedeutet jedenfalls höchste Gefahr. Gelingt es nicht, im Harn die Diagnose zu stellen, ist der Nachweis durch Leberbiopsie mit Erkennung der Rotfluoreszenz leicht $\mathrm{zu}$ führen. Uroporphyrin-Untersuchung kann aber auch im Duodenalsaft und bei der Prophyria cutanea tarda im Stuhl vorgenommen werden. Im Beginn der Porphyria hepatica sieht man kleine Inselchen von Rotfluoreszenz im Leberstückchen als hepatische partielle Porphyrie, bei stärkerem Befall werden sie immer zahlreicher und konfluieren schließlich.

Eine solche Feststellung ist jedoch von großer klinischer Bedeutung und bedarf der entsprechenden therapeutischen Einstellung. Unsere moderne Zeit bringt zusätzliche Gefahren für die Menschen mit sich, deren Kontrolle von ärztlicher Seite auch auf dem Gebiete der Hämsynthese erforderlich ist. Die Häufung von Porphyrien als toxische Porphyrien durch Alkohol, Medikamente und Vergiftungen kann und muß vermieden werden. Hierfür ist die Kontrolle der Hämsynthese durch den Arzt und ein Porphyrinlaboratorium unumgänglich und muß in Zukunft noch verbessert werden.

\section{Literatur}

1. GüNtrakr, H., S.ber. Niederrhein. Ges. Nat.-Heilk., Sitzung 13. III. 1911. - 2. GüNther, H., Dtsch. Arch. klin. Med. 134, 257 (1920). - 3. Kämmerer, H., Verh. Dtsch. Ges. inn. Med. 45, 28 (1933). - 4. Schimid, R., S. Schwartz und C. J. Watson, Acta haemat, 10, 150 (1953). - 5. BrugsCh, J. und A. BIENENGRÄBER, Zschr. inn. Med., Leipzig 10, 1117 (1955). - 6. BRUGSCH,
J., Zschr. inn. Med., Leipzig 11, 5 (1956). - 7. Eppinger, H., Die hepatolienalen Erkrankungen Springer, Berlin (1920). 8. Waldenström, J., Acta med. Scand. Suppl. 90, 380 (1937). 9. Brugsch, J., Porphyrine, 2. Aufl. J. A. Barth, Leipzig (1959). 10. Brugsch, J., Zschr. inn. Med., Leipzig 4, 253 (1949). 